\title{
Análisis bibliométrico de la producción científica peruana en cardiología y medicina cardiovascular
}

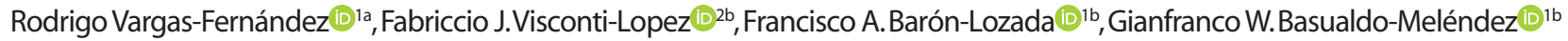

Recibido: 14 de septiembre de 2021
Aceptado: 28 de septiembre de 2021
Filiación de los autores
Universidad Científica del Sur.
Lima, Perú.
Universidad Peruana de Ciencias
Aplicadas. Lima, Perú.
a Médico cirujano
b Estudiante de medicina
*Correspondencia
Rodrigo Vargas-Fernández
Panamericana Sur km 19, Villa el
Salvador, Lima, Perú
(00511) 6106400
Correo
jvargasf@cientifica.edu.pe
Financiamiento
Autofinanciado.
Conflictos de interés
Los autores declaran no tener
conflictos de interés.
Citar como:
Vargas-Fernández R, Visconti-Lopez
FJ, Barón-Lozada FA, Basualdo-
Meléndez GW. Análisis bibliométrico
de la producción científica
peruana en cardiología y medicina
cardiovascular. Arch Peru Cardiol Cir
Cardiovasc. 2021;2(3):167-174. doi:
10.47487/apcyccv.v2i3.157.

\section{RESUMEN}

Objetivo. Determinar las características y la tendencia de los artículos publicados sobre cardiología y medicina cardiovascular en el contexto peruano, y comprender cómo ha cambiado a lo largo de los años. Materiales y métodos. Se realizó un estudio bibliométrico de artículos originales publicados hasta el año 2020 por autores peruanos en revistas indexadas bajo la categoría «Cardiac \& Cardiovascular Systems» en Web of Science (WOS). Se incluyeron los artículos según los criterios de selección en el aplicativo web Rayyan y se realizó el análisis bibliométrico mediante el paquete Bibliometrix en el lenguaje de programación R y VOSviewer. Resultados. Se incluyeron 159 artículos publicados, y se observó un incremento del número de publicaciones desde 2015. El artículo más citado fue un ensayo clínico realizado por Fitchett et al. y publicado en 2016. Miranda JJ fue el autor peruano con mayor número artículos publicados seguido de Hernández AV y Málaga G. La filiación institucional con mayor número de artículos originales fue la Universidad Peruana Cayetano Heredia. En cuanto a los términos o palabras clave, se encontró que la mayor cantidad de estudios publicados contaban con términos relacionados con la epidemiología, mientras que en los artículos más recientes, los términos se relacionaron con desenlaces o intervenciones específicas que son utilizados en estudios clínicos. Conclusiones. Se encontró un incremento en los últimos cinco años en la producción científica sobre cardiología y medicina cardiovascular de autores con filiación institucional peruana, con una mayor producción de la Universidad Peruana Cayetano Heredia. La revista con mayores publicaciones de autores con filiación institucional peruana sobre cardiología y medicina cardiovascular fue Circulation, donde también se encontraron dos de los artículos con filiación institucional peruana más citados.

Palabras clave: Cardiología; Enfermedades cardiovasculares; Bibliometría; Publicaciones; Perú (fuente: DeCS BIREME).

\section{ABSTRACT}

\section{Bibliometric analysis of peruvian scientific production in cardiology and cardiovascular medicine}

\begin{abstract}
Objective. To determine the characteristics and trend of the articles published on cardiology and cardiovascular medicine in the Peruvian context, and to understand how it has changed over the years. Materials and methods. A bibliometric study of original articles published up to 2020 by Peruvian authors in journals indexed under the category "Cardiac \& Cardiovascular Systems" in Web of Science (WOS) was performed. The articles were included according to the selection criteria in the Rayyan web application and the bibliometric analysis was performed using the Bibliometrix package in the R programming language and VOSviewer. Results. A total of 159 published articles were included, and an increase in the number of publications since 2015 was observed. The most cited article was a clinical trial by Fitchett et al. and published in 2016. Miranda JJ was the Peruvian author with the highest number of published articles followed by Hernández AV and Málaga G. The institutional affiliation with the highest number of original articles was Universidad Peruana Cayetano Heredia. Regarding the terms or keywords, it was found that most of the published studies had terms related to epidemiology, while in the most recent articles, the terms were related to outcomes or specific interventions that are used in clinical studies. Conclusions. In the last five years, there has been an increase in the scientific production on cardiology and cardiovascular medicine by authors with Peruvian institutional affiliation, with a greater production from the Universidad Peruana Cayetano Heredia. The journal with the highest number of publications by authors with Peruvian institutional affiliation on cardiology and cardiovascular medicine was Circulation, where two of the most cited articles with Peruvian institutional affiliation were also found.
\end{abstract}

Keywords: Cardiology; Cardiovascular diseases; Bibliometric; Publications; Peru (source: MeSH NLM). 


\section{Introducción}

Las enfermedades cardiovasculares (ECV) son la principal causa de muerte y uno de los mayores contribuyentes de morbimortalidad en el mundo (1). Se estima que los casos prevalentes de ECV se han duplicado entre 1990 y 2019, superando los 500 millones a nivel global ${ }^{(1)}$. Asimismo, la carga de enfermedad y mortalidad por ECV se ha incrementado significativamente durante el mismo periodo, donde los años de vida perdidos por discapacidad se han duplicado de 17,7 a 34,4 millones, y el número de muertes por ECV ha aumentado en más de seis millones entre 1990 y $2019^{(1)}$. Estas cifras son mayores en países de medianos y bajos ingresos ${ }^{(2)}$, donde ocurre casi el $80 \%$ de muertes ${ }^{(2)}$. En este sentido, la importancia de la investigación en cardiología y ECV radica en el incremento constante de la morbimortalidad por estas patologías que han generado gran preocupación en los sistemas sanitarios de todo el mundo, sobre todo en países con recursos limitados en salud ${ }^{(3)}$.

La investigación científica es un mecanismo para estudiar y mejorar la salud de las poblaciones, y frenar el incremento de la carga de las enfermedades crónicas no transmisibles mediante los principios de la medicina basada en evidencia, elaboración de políticas públicas y asignación de fondos nacionales e internacionales ${ }^{(4)}$. Sin embargo, los países de medianos y bajos ingresos tienen los mayores desafíos debido a la escasez de información basada en la evidencia, a la calidad y por restricciones en el uso de bases de datos en salud y la asignación de recursos para la investigación ${ }^{(4-6)}$ lo cual genera una baja producción científica sobre ECV ${ }^{(7)}$. Por ello, los estudios bibliométricos son ideales para abordar esta problemática, puesto que proporcionan información sobre los resultados del proceso investigador, el volumen, la evolución, la visibilidad y la estructura, para así valorar la actividad científica y el impacto tanto de la investigación como de las fuentes ${ }^{(8)}$.

Existen pocos registros de análisis bibliométricos sobre la producción científica en cardiología y salud cardiovascular, la mayoría comprenden autores de la Unión Europea, Estados Unidos, Asia y Cuba ${ }^{(9,10)}$. Actualmente, no se tiene registro de cuál ha sido la producción científica peruana sobre cardiología y salud cardiovascular. Por tal motivo, el objetivo de este estudio es determinar las características y la tendencia de los artículos publicados sobre cardiología y medicina cardiovascular en el contexto peruano, y comprender cómo ha cambiado a lo largo de los años.

\section{Materiales y métodos}

Se realizó un análisis bibliométrico de artículos originales publicados por autores peruanos en revistas indexadas bajo la categoría «Cardiac \& Cardiovascular Systems» en Web of Science (WOS).

\section{Estrategia de búsqueda}

La estrategia de búsqueda incluyó los términos «Peru OR Peruvian*» que se buscaron en todos los campos de WOS («All Fields») en la categoría "Cardiac \& Cardiovascular Systems», y se seleccionaron solo los tipos de documentos bajo la clasificación de «Articles». La búsqueda se llevó a cabo el 11 de septiembre de 2021. Se consideró incluir únicamente artículos publicados hasta el 2020 debido a que el año 2021 está aún en curso. No se consideró aplicar restricciones de idioma.

\section{Selección de artículos}

Los metadatos de los registros completos identificados en la búsqueda fueron descargados como archivo .ciw. Seguidamente, se importaron al aplicativo web Rayyan, donde dos autores (FABL y GWBM) de forma independiente, revisaron y seleccionaron los títulos, resúmenes y autores de cada uno de los registros que cumplían con el criterio de inclusión: artículo original publicado hasta el año 2020 con al menos un autor con filiación de una institución peruana. Los conflictos en la selección fueron solucionados por consenso entre los revisores (FABL y GWBM). De cada uno de los registros que no fueron incluidos, se extrajo el «Accession Number» de WOS para excluirlos de la búsqueda inicial y obtener los registros completos finales para el análisis bibliométrico.

\section{Análisis bibliométrico}

La obtención de índices bibliométricos se realizó mediante el paquete Bibliometrix en el lenguaje de programación $\mathrm{R}{ }^{(11)}$. Asimismo, se empleó el software VOSviewer versión 1.6.17 (Leiden University, Leiden, The Netherlands) ${ }^{(12)}$ para elaborar redes bibliométricas basadas en la coautoría, empleando información sobre los nombres de los autores, filiaciones institucionales y palabras clave de los registros recuperados. Previamente al análisis de redes, se realizó una estandarización de los datos de forma manual en el campo de autor, filiación institucional y keywords para eliminar redundancias e incoherencias mediante la creación de tesauros en formato .txt bajo el formato de dos columnas (label y replace by) según lo establecido en el manual del software VOSviewer versión 1.6.17 ${ }^{(13)}$. Asimismo, se utilizó Microsoft Excel para elaborar tablas y gráficos.

Se reportó el total de artículos, total de revistas, total de citas, total de citaciones, número de artículos publicados anualmente, diez artículos con mayor número de citaciones, revistas con mayor número de publicaciones, red de coautoría según autores, red de coautoría según filiaciones institucionales y red de coocurrencia 
de palabras clave. Los análisis de redes se realizaron con el método de conteo fractional counting, en documentos con un máximo de 50 autores, un mínimo de 1 documento por autor, método de normalización de asociación, repulsión de los nodos de 2, atracción de los nodos de 1, resolución de los cluster de 1, mínimo del tamaño del cluster de 1, peso según el número de artículos, puntuación para la temporalidad según el promedio de publicación al año y solo en el caso de la red de coocurrencia de palabras clave se utilizó un umbral para la coocurrencia en títulos y resúmenes de cinco menciones. Los valores de los nodos fueron establecidos según las recomendaciones del manual del software VOSviewer versión 1.6.17 ${ }^{(13)}$.

\section{Consideraciones éticas}

No se solicitó la aprobación del estudio a un comité de ética por tratarse de un análisis de publicaciones en bases de datos bibliográficas.

\section{Resultados}

Se encontraron 174 artículos; de estos, se excluyeron 15 basados en el título, resumen y autoría de cada artículo por medio del aplicativo web Rayyan. La base final fueron 159 artículos, de un total de 63 revistas. El promedio anual de artículos publicados fue de 3,61; el mayor incremento en artículos fue en el año 2015, y una mayor producción en el año 2019 y 2020 (26 documentos); asimismo, se observó una tendencia polinómica de cuarto orden en las publicaciones entre 1976 y el 2020 con un R2 de 0,88 (Figura 1).
En cuanto a los artículos seleccionados, en conjunto tuvieron un total de 4854 citas, con un promedio de 30,53 referencias por documento. El artículo más citado fue un ensayo clínico realizado por Fitchett et al. publicado en la revista de European Heart Journal en $2016^{(14)}$. Este artículo, titulado Heart failure outcomes with empagliflozin in patients with type 2 diabetes at high cardiovascular risk: results of the EMPA-REG OUTCOME ${ }^{\circledR}$ trial, tuvo un total de 568 citas, seguido del artículo de Packer et al. (408 citas) publicado en $2015^{(15)}$ y Penaloza y Arias-Stella. (341 citas) publicado en $2007^{(16)}$. La revista Global Heart fue donde más se publicaron artículos de autores peruanos sobre cardiología y medicina cardiovascular $(n=13)$, seguido de Circulation $(n=12)$ y Journal of the American Heart Association $(n=10)($ Tabla 1).

En cuanto a los autores peruanos, Miranda JJ, con filiación en la Universidad Peruana Cayetano Heredia, fue el autor con mayor número de artículos publicados en el área de cardiología, y con una mayor presencia a partir del año 2014; a diferencia de los autores Hernández AV (con filiaciones peruanas en la Universidad San Ignacio de Loyola y Universidad Peruana de Ciencias Aplicadas) y Málaga G (con filiaciones peruanas en la Universidad Peruana Cayetano Heredia y Hospital Nacional Cayetano Heredia) quienes tuvieron mayor presencia alrededor del año 2018 según el promedio de publicación al año. Además, se observa que Lemor A es el autor que más artículos ha publicado recientemente (Figura 2).

Hubo 749 filiaciones, de las cuales, la Universidad Peruana Cayetano Heredia fue la más reportada, seguida de la Johns Hopkins University; no obstante, se observa que la filiación del Hospital Nacional Dos de Mayo fue la que estuvo

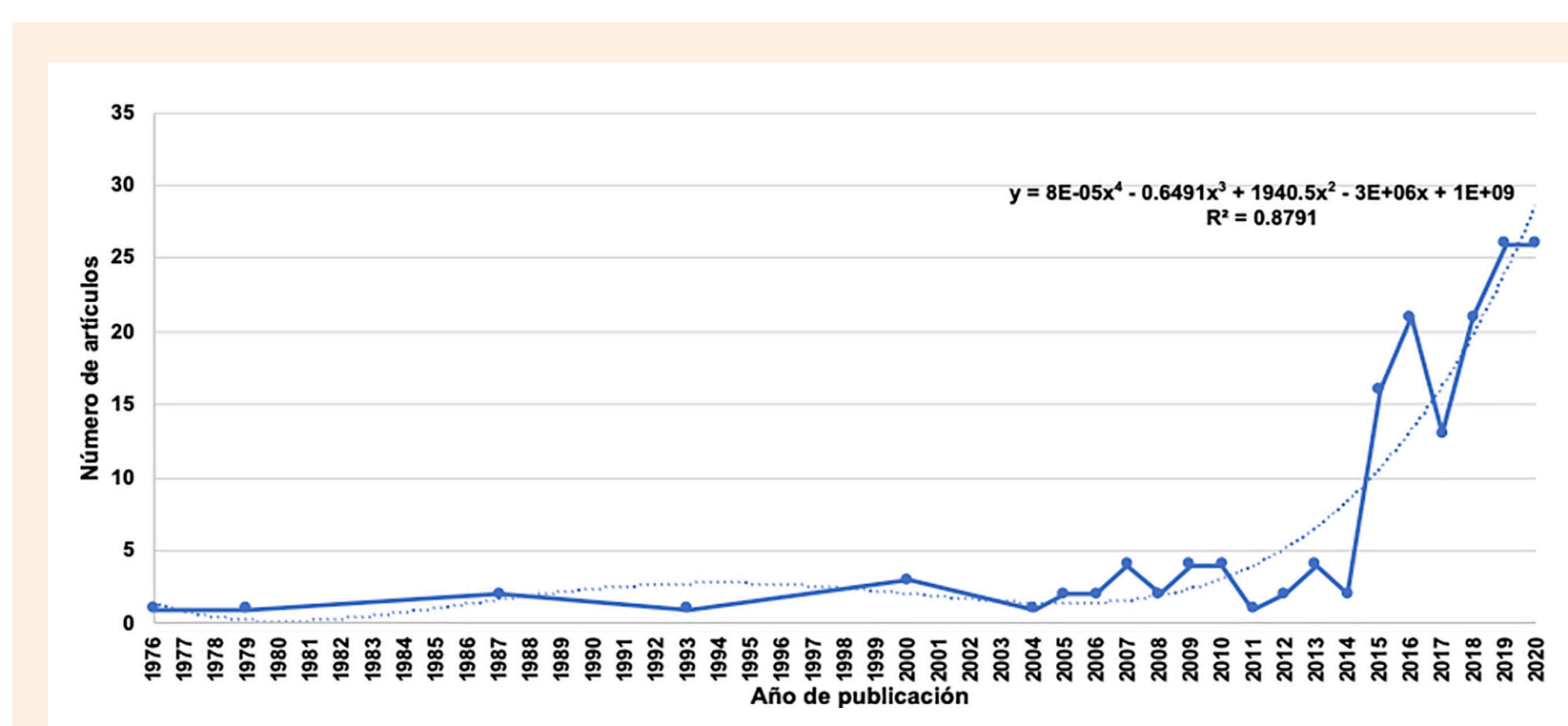

Figura 1. Tendencia de la publicación de artículos en cardiología y medicina cardiovascular de autores peruanos en Web of Science, desde 1976 hasta 2020 
Tabla 1. Artículos con mayor número de citas y revistas con mayor número de artículos en cardiología y medicina cardiovascular con autores peruanos

\begin{tabular}{|c|c|}
\hline Característica & Total \\
\hline Artículo & N. ${ }^{\circ}$ citas \\
\hline $\begin{array}{l}\text { Heart failure outcomes with empagliflozin in patients with type } 2 \text { diabetes at high cardiovascular risk: } \\
\text { results of the EMPA-REG OUTCOME } \text { trial }^{a}\end{array}$ & 568 \\
\hline $\begin{array}{l}\text { Angiotensin receptor neprilysin inhibition compared with enalapril on the risk of clinical progression in } \\
\text { surviving patients with heart failure }{ }^{b}\end{array}$ & 408 \\
\hline $\begin{array}{l}\text { The heart and pulmonary circulation at high altitudes: healthy highlanders and chronic mountain } \\
\text { sickness }{ }^{c}\end{array}$ & 341 \\
\hline $\begin{array}{l}\text { Empagliflozin and Clinical Outcomes in Patients With Type } 2 \text { Diabetes Mellitus, Established Cardiovas- } \\
\text { cular Disease, and Chronic Kidney Disease }^{d}\end{array}$ & 216 \\
\hline $\begin{array}{l}\text { Effects of empagliflozin on risk for cardiovascular death and heart failure hospitalization across the } \\
\text { spectrum of heart failure risk in the EMPA-REG OUTCOME } \text { triale }^{\text {e }}\end{array}$ & 145 \\
\hline $\begin{array}{l}\text { International collaborative project to compare and monitor the nutritional composition of processed } \\
\text { foods }^{f}\end{array}$ & 109 \\
\hline Effect of Alirocumab on Lipoprotein(a) and Cardiovascular Risk After Acute Coronary Syndrome ${ }^{g}$ & 94 \\
\hline Alirocumab Reduces Total Nonfatal Cardiovascular and Fatal Events: The ODYSSEY OUTCOMES Trial ${ }^{\mathrm{h}}$ & 88 \\
\hline $\begin{array}{l}\text { Femoral and Carotid Subclinical Atherosclerosis Association With Risk Factors and Coronary Calcium: } \\
\text { The AWHS Studyi }\end{array}$ & 87 \\
\hline Placebo-controlled study of lisinopril in congestive heart failure: a multicentre studyj & 81 \\
\hline Revista (cuartil SJR) & N. ${ }^{\circ}$ artículos \\
\hline Global Heart (Q1) & 13 \\
\hline Circulation (Q1) & 12 \\
\hline Journal of The American Heart Association (Q1) & 10 \\
\hline American Journal of Cardiology (Q1) & 9 \\
\hline American Journal of Physiology Heart and Circulatory Physiology (Q1) & 8 \\
\hline European Heart Journal (Q1) & 8 \\
\hline Archivos de Cardiología de México (Q4) & 7 \\
\hline Journal of The American College of Cardiology (Q1) & 7 \\
\hline Heart (Q1) & 6 \\
\hline International Journal of Cardiology (Q1) & 6 \\
\hline
\end{tabular}

Se utilizo el Scimago Journal \& Country Rank para colocar el cuartil de cada revista.

a 10.1093/eurheartj/ehv728; b 10.1161/CIRCULATIONAHA.114.013748; c 10.1161/CIRCULATIONAHA.106.624544; d 10.1161/CIRCULATIONAHA.117.028268; e 10.1093/

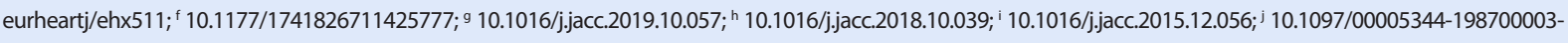
00021

presente en la mayor cantidad de artículos alrededor del año 2005 (Figura 3).

En lo que respecta a las palabras clave, se identificaron un total de 953 términos. Como se observa en la Figura 4, las palabras clave predominantes en el 2014 fueron hypoxia, exercise, coronary-heart-disease y cholesterol; sin embargo, las palabras clave que están en un mayor número de artículos son mortality y myocardial infarction. Asimismo, se observa que los artículos más recientes utilizan nuevas palabras clave, como acute coronary syndrome, alirocumab y cardiopulmonary resuscitation.

\section{Discusión}

El presente estudio buscó determinar la producción científica peruana en cardiología y medicina cardiovascular hasta 2020 en WOS. Como resultado, se evidencia un incremento en el número de artículos originales sobre cardiología y medicina cardiovascular por los autores con filiación institucional peruana a partir de 2015, con la mayor producción de artículos en 2019 y 2020. Se identificaron a los autores y las instituciones con sus respectivas redes de colaboración que lideran la investigación 


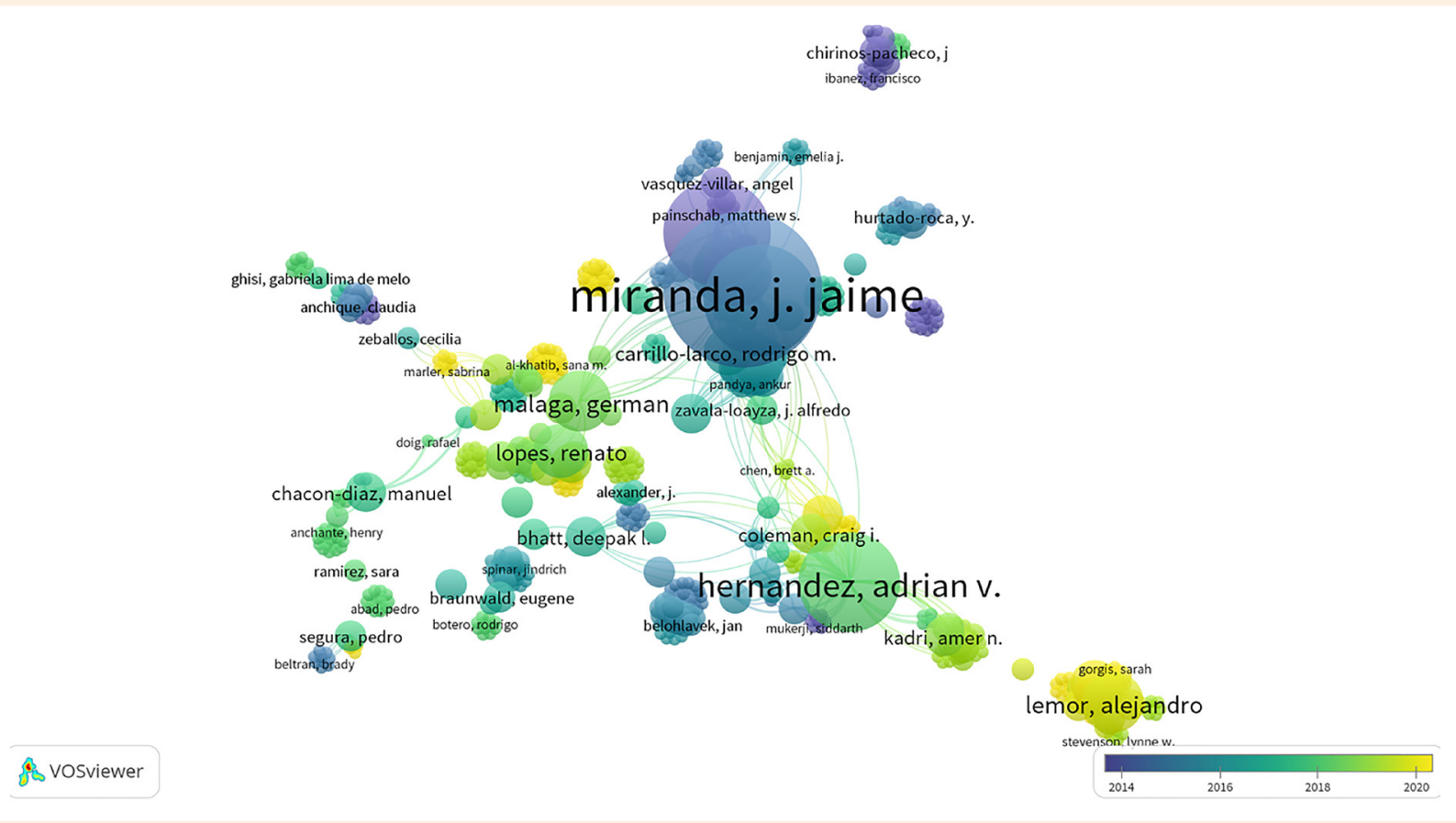

Figura 2. Mapa de visualización de la red de autores.

Los diferentes colores representan el promedio de publicación al año. Los círculos grandes indican los autores con mayor número de publicaciones.

sobre cardiología y medicina cardiovascular, principales revistas científicas donde fueron publicados los artículos originales y las palabras clave más estudiadas en los últimos años.

Con relación a la producción científica peruana sobre cardiología y medicina cardiovascular, se evidenció un aumento del número de artículos originales publicados a partir de 2015, donde se observa que la producción se triplicó comparado con la producción observada en años anteriores. Asimismo, se debe considerar que la mayor producción de artículos originales se observó en 2019 y 2020, alcanzando un total de 159 artículos

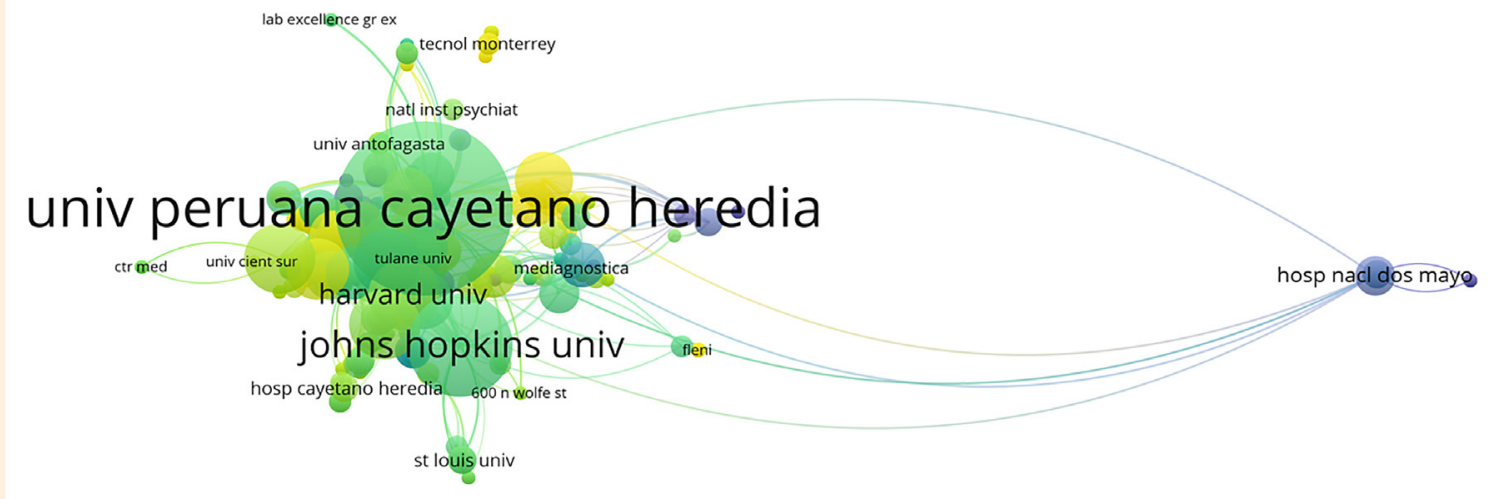

f

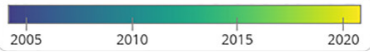

Figura 3. Mapa de visualización de la red de filiaciones

Los diferentes colores representan el promedio de publicación al año. Los círculos grandes indican las filiaciones de alta frecuencia. 


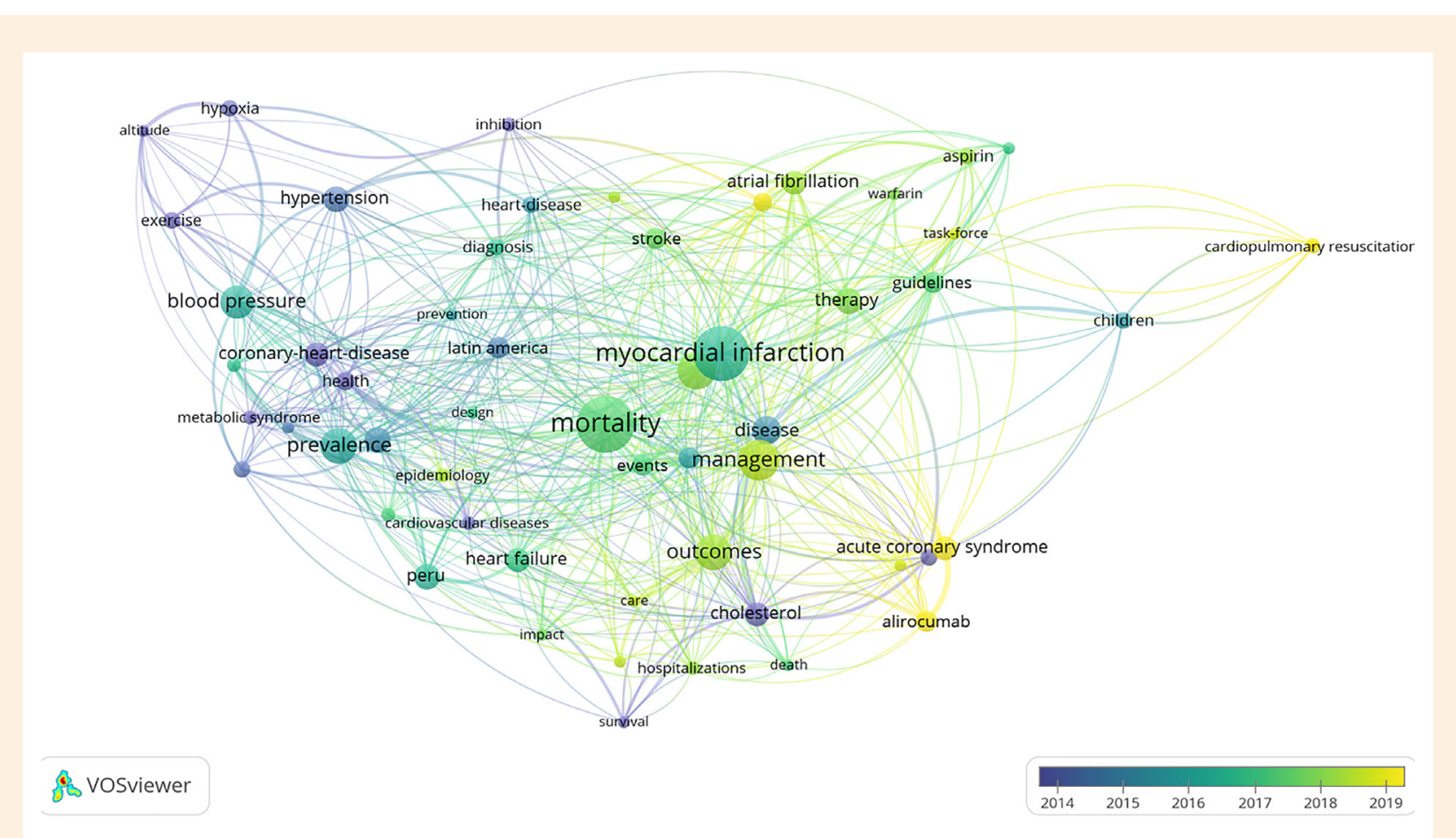

Figura 4. Mapa de visualización de la red de palabras clave.

Los diferentes colores representan el promedio de publicación al año. Los círculos grandes indican las palabras clave de alta frecuencia.

publicados entre 1976 y 2020. En contraste con estos hallazgos, se debe señalar que la producción de la investigación cardiovascular global aumentó de forma constante en la última década, con un incremento de más del $40 \%$ en el número de publicaciones en 2017 comparado con 2008, y un aumento significativo a partir de 2013. Sin embargo, los países de mayores ingresos tuvieron la mayor contribución a la investigación cardiovascular global (con más del $80 \%$ de la producción científica) comparado con los países de medianos y altos ingresos $(16,1 \%)$, medianos y bajos ingresos $(2,6 \%)$ y bajos ingresos $(0,2 \%){ }^{(4)}$. La región de Latinoamérica y el Caribe publicó el $4 \%$ de artículos comparado con los países de altos ingresos como Estados Unidos y Canadá; donde Brasil, Argentina, México y Chile tienen el mayor número de publicaciones sobre medicina cardiovascular, mientras que Perú se encontraba dentro de los niveles bajos de publicaciones por arriba de países de Centroamérica y Bolivia en $2008^{(17,18)}$. Este aumento de la producción científica peruana es importante para la toma de decisiones y desarrollo de estrategias para el control de enfermedades cardiovasculares, además, se requiere que este incremento sea constante en el tiempo para lograr mayores avances en la investigación sobre medicina cardiovascular, sobre todo por los cambios en el perfil epidemiológico que ha generado un aumento de la prevalencia de las enfermedades crónicas no transmisibles ${ }^{(19)}$.

En cuanto a los artículos con mayor número de citas que tienen un autor con filiación institucional peruana, se observa que el artículo más citado fue un ensayo clínico (ECA) de fase III realizado por Fitchett et al. y publicado en el 2016, con un total de 568 citas $^{(14)}$, seguido de un ECA de fase III realizado por Packer et al. en 2014 (con 408 citas) ${ }^{(15)}$, y el artículo de revisión realizado por Penaloza et al. publicado en 2007 (con 341 citas) ${ }^{(16)}$. Además, en cuanto a las principales revistas científicas donde fueron publicados los artículos originales sobre cardiología y medicina cardiovascular, se encontró que la revista Global Heart fue donde más se publicaron artículos de autores peruanos sobre cardiología y medicina cardiovascular, seguida de Circulation y la Journal of the American Heart Association. Estos hallazgos son similares a lo reportado por Opthof, donde observó que las cuatro revistas más importantes y citadas sobre cardiología y medicina cardiovascular fueron la European Heart Journal, Circulation, Journal of the American College of Cardiology and Circulation Research ${ }^{(20)}$. Así, los ECA con mayor número de citas son multicéntricos y realizados en diferentes países, que frecuentemente se reportan en revistas de alto impacto como las mencionadas previamente.

Cabe precisar que de los tres artículos publicados con mayor número de citas, el artículo realizado por Penaloza y Arias-Stella, titulado The Heart and Pulmonary Circulation at High Altitudes, fue el único estudio realizado solo por autores con filiación institucional peruana ${ }^{(16)}$. Este artículo fue una revisión de la literatura sobre la fisiología, patología, patogénesis y características clínicas del corazón y circulación pulmonar en montañeses sanos y en pacientes con mal de montaña crónico; 
los autores concluyeron que los estudios pioneros realizados por investigadores peruanos contribuyeron a dilucidar la patogénesis de la hipertensión pulmonar de altura y la hipertrofia ventricular derecha en personas sanas nacidas y que viven en gran altitud. Este hallazgo refleja el interés de la altitud en la investigación peruana, debido a que el Perú es un país andino con regiones de gran altitud, en las cuales, se estima, viven más de cinco millones de personas ${ }^{(21)}$.

Con relación a los autores con filiación institucional peruana, se encontró que Miranda JJ fue el autor con mayor número artículos publicados en el área de cardiología, y con una mayor presencia a partir del año 2014, a diferencia de los autores Hernández AV y Málaga G, quienes tuvieron mayor presencia alrededor del año 2018, según el promedio de publicación al año. Además, se observa que Lemor A tuvo una mayor producción de artículos en el último año. En el Perú, solo existe un instituto especializado en patologías cardiovasculares y pertenece al Seguro Social de Salud (EsSalud) del cual se espera que tenga una importante producción ${ }^{(22)}$. De esta institución, Chacón-Diaz M es el autor con mayor número de publicaciones.

En cuanto a las filiaciones institucionales, se encontró que la institución que tiene mayor publicación de artículos originales sobre cardiología y medicina cardiovascular es la Universidad Peruana Cayetano Heredia. Esto concuerda con lo reportado sobre Miranda JJ, quién es autor de estudios epidemiológicos como CRONICAS y PERU MIGRANT, los cuales son estudios de cohorte sobre enfermedades crónicas no transmisibles que pertenecen a dicha universidad y que avalan la producción sobre cardiología y enfermedades cardiovasculares de esta institución ${ }^{(23)}$. La Universidad Peruana Cayetano Heredia es la institución con mayor producción científica en el territorio peruano, pues un tercio de las publicaciones científicas entre 1997 y 2016, están vinculadas con esta institución ${ }^{(24)}$. Asimismo, otras instituciones educativas internacionales como la Universidad de Johns Hopkins y la Universidad de Harvard ocuparon posiciones de liderazgo en la producción científica peruana sobre cardiología y medicina cardiovascular como parte de las redes de colaboración. Este hallazgo podría estar relacionado con la Universidad Peruana Cayetano Heredia, debido a que los investigadores que pertenecen a esta institución han mantenido alianzas y financiación internacionales, lo que se refleja en la coautoría de publicaciones, donde más de la mitad corresponden a artículos que fueron escritos y publicados con profesionales de instituciones de Estados Unidos (24). Adicionalmente, destacaron otras instituciones como el Hospital Nacional
Dos de Mayo, el cual es un hospital emblemático y uno de los más antiguos del territorio peruano, y el Hospital Cayetano Heredia, debido a la dependencia institucional existente con la Universidad Peruana Cayetano Heredia.

Por último, en cuanto a la coocurrencia de términos, se observa que los términos utilizados con mayor antigüedad estaban relacionados con altitud, ejercicio, hipoxia, colesterol y enfermedad cardiovascular, los cuales son términos relacionados con factores de riesgo o de exposición que se asocian con enfermedades cardíacas y cardiovasculares (25-27). En años más recientes, los términos que se encuentran en un mayor número de artículos son mortalidad, infarto de miocardio, resultados y manejo; términos que están vinculados con la epidemiología (28), lo que refleja el interés de los autores con mayor producción científica sobre la epidemiología (29). Por último, los términos utilizados en 2019 fueron síndrome coronario agudo, alirocumab y resucitación cardiopulmonar, lo que pone de manifiesto la importancia de los desenlaces específicos o intervenciones en los estudios a nivel clínico sobre patologías cardiacas y cardiovasculares, que han crecido en tamaño, alcance y complejidad por la aparición de nuevos medicamentos o intervenciones ${ }^{(30)}$.

Dentro de las limitaciones del estudio, se debe mencionar que un análisis bibliométrico depende de la disponibilidad de los datos de los artículos recuperados con la estrategia de búsqueda. Asimismo, se debe considerar que la búsqueda se realizó en una sola base de datos (WOS), por lo que no se incluiría la producción peruana sobre cardiología y medicina cardiovascular en otras bases de datos bibliográficas como Scopus, Medline y SciELO. A pesar de estas limitaciones, consideramos que el estudio de la producción científica peruana sobre cardiología y medicina cardiovascular en una de las bases de datos bibliográficas más importantes como es WOS, permite evidenciar cuales son los avances en conocimiento sobre estas áreas de investigación en el país y como ha progresado la producción científica en los investigadores peruanos.

En conclusión, se encontró un incremento en la producción científica sobre cardiología y medicina cardiovascular de autores con filiación institucional peruana, con una mayor producción de la Universidad Peruana Cayetano Heredia. La revista con mayores publicaciones de autores con filiación institucional peruana sobre cardiología y medicina cardiovascular fue Circulation, donde también se encontraron dos de los artículos con filiación institucional peruana más citados. Los términos utilizados en los artículos más recientes se relacionan con desenlaces o intervenciones específicas que actúan en patologías cardíacas y cardiovasculares. 


\section{Contribución de los autores}

FABL y GWBM revisaron que los registros obtenidos de la búsqueda cumplan con los criterios de inclusión. Todos los autores participaron en la interpretación de los datos, redacción del manuscrito, aprobaron la versión final y son responsables por los contenidos.

\section{Agradecimientos}

Al Dr. Akram Hernández por su apoyo en la búsqueda bibliográfica y el análisis bibliométrico, y que además agradece a la Universidad de Chile por brindar acceso a Web of Science a sus alumnos y egresados.

\section{Referencias bibliográficas}

1. Roth GA, Mensah GA, Johnson CO, Addolorato G, Ammirati E, Baddour LM, et al. Global Burden of Cardiovascular Diseases and Risk Factors, 1990-2019: Update from the GBD 2019 Study. J Am Coll Cardiol. 2020;76(25):2982-3021. doi: 10.1016/j.jacc.2020.11.010.

2. Prabhakaran D, Anand S, Watkins D, Gaziano T, Wu Y, Mbanya JC, et al. Cardiovascular, respiratory, and related disorders: key messages from Disease Control Priorities, 3rd edition. Lancet. 2018;391(10126):122436. doi: 10.1016/S0140-6736(17)32471-6.

3. Dzau VJ, Balatbat CA. Cardiovascular Research and the National Academy of Medicine: Advancing Progress in Science and Medicine. Cir Res. 6 de enero de 2017;120(1):23-6.

4. Qureshi NQ, Mufarrih SH, Bloomfield GS, Tariq W, Almas A, Mokdad $\mathrm{AH}$, et al. Disparities in Cardiovascular Research Output and Disease Outcomes among High-, Middle- and Low-Income Countries - An Analysis of Global Cardiovascular Publications over the Last Decade (2008-2017). Glob Heart. 2021;16(1):4. doi: 10.5334/gh.815.

5. Goyet S, Touch S, Ir P, SamAn S, FassierT, Frutos R, et al. Gaps between research and public health priorities in low-income countries: evidence from a systematic literature review focused on Cambodia. Implement Sci. 2015;10(1):32. doi: 10.1186/s13012-015-0217-1.

6. Albarqouni L, Elessi K, Abu-Rmeileh NME. A comparison between health research output and burden of disease in Arab countries: evidence from Palestine. Health Res Policy and Sys. 2018;16(1):25. doi: 10.1186/s12961-018-0302-4

7. Vidyasagar D. Global notes: the $10 / 90$ gap disparities in global health research. J Perinatol. 2006;26(1):55-6. doi: 10.1038/sj.jp.7211402.

8. Camps D. Limitaciones de los indicadores bibliométricos en la evaluación de la actividad científica biomédica. Colombia Médica. 2008;39(1):74-9.

9. Devos P, Menard J. Bibliometric analysis of research relating to hypertension reported over the period 1997-2016. J Hypertens. 2019;37(11):2116-22. doi: 10.1097/HJH.0000000000002143.

10. Vitón-Castillo AA, Díaz-Samada RE, Pérez Álvarez DA, CasínRodríguez SM, Casabella Martínez S, Vitón-Castillo AA, et al. Análisis bibliométrico de la producción científica sobre cardiología publicada en las revistas científicas estudiantiles cubanas (2014-2018). CorSalud. 2019;11(1):39-45.

11. Aria M, Cuccurullo C. Bibliometrix : An R-tool for comprehensive science mapping analysis. J Informetr. 2017;11(4):959-75. doi: 10.1016/j.joi.2017.08.007.

12. Van Eck NJ, Waltman L. Software survey: VOSviewer, a computer program for bibliometric mapping. Scientometrics. agosto de 2010;84(2):523-38. doi: 10.1007/s11192-009-0146-3.

13. Van Eck NJ, Waltman L. VOSviewer Manual [Internet]. Leiden: Centre for Science and Technology Studies; 2021 [citado 14 de septiembre de 2021]. Disponible en: https://www.vosviewer.com/ documentation/Manual_VOSviewer_1.6.17.pdf

14. Fitchett D, Zinman B, Wanner C, Lachin JM, Hantel S, Salsali A, etal. Heart failure outcomes with empagliflozin in patients with type 2 diabetes at high cardiovascular risk: results of the EMPA-REG OUTCOME ${ }^{\circledR}$ trial. Eur Heart J. 2016;37(19):1526-34. doi: 10.1093/eurheartj/ehv728.

15. Packer M, McMurray JJV, Desai AS, Gong J, Lefkowitz MP, Rizkala $A R$, et al. Angiotensin receptor neprilysin inhibition compared with enalapril on the risk of clinical progression in surviving patients with heart failure. Circulation. 2015;131(1):54-61. doi: 10.1161/ CIRCULATIONAHA.114.013748.

16. Penaloza D, Arias-Stella J. The heart and pulmonary circulation at high altitudes: healthy highlanders and chronic mountain sickness. Circulation. 2007;115(9):1132-46. doi: 10.1161/ CIRCULATIONAHA.106.624544.

17. Jahangir E, Comandé D, Rubinstein A. Cardiovascular disease research in Latin America: a comparative bibliometric analysis. World J Cardiol. 2011;3(12):383-7. doi: 10.4330/wjc.v3.i12.383.

18. Colantonio LD, Baldridge AS, Huffman MD, Bloomfield GS, Prabhakaran D. Cardiovascular research publications from Latin America between 1999 and 2008. A bibliometric study. Arq Bras Cardiol. 2015;104(1):5-15. doi: 10.5935/abc.20140213.

19. Samet JM, Brownson RC. Epidemiology in a Changing World. American Journal of Preventive Medicine. 2014;47(5):S383-5.

20. Opthof T. Comparison of the Impact Factors of the Most-Cited Cardiovascular Journals. Circ Res. 7 de junio de 2019;124(12):1718-24.

21. Tremblay JC, Ainslie PN. Global and country-level estimates of human population at high altitude. Proc Natl Acad Sci USA. 2021;118(18):e2102463118. doi: 10.1073/pnas.2102463118.

22. Alarcón C, Cortez-Soto AG, Romero-Cerdan A, Benites-Bullón A, Altamirano-Farfá EF, Pino-Zavaleta DA, et al. Producción científica y redes de colaboración en la Seguridad Social del Perú (EsSalud): Análisis bibliométrico 2008-2020. Cinta de moebio. 2021;14(2):14554. doi: 10.35434/rcmhnaaa.2021.142.1030.

23. Del Castillo-Fernández D, Condorena AB, Landeo PV, García LS, Ortiz $A B$, Miranda J. Avances en la investigación de enfermedades crónicas no transmisibles en el Perú. An Fac med; 2020;81(4):444-452. doi: 10.15381/anales.v81i4.18798.

24. Glass RI, Garcia PJ, Belter CW, Livinski AA, Leon-Velarde F. Rapid growth of biomedical research in Peru. Lancet Glob Health. julio de 2018;6(7):e728-9. doi: 10.1016/S2214-109X(18)30234-1.

25. Carson JAS, Lichtenstein AH, Anderson CAM, Appel LJ, Kris-Etherton PM, Meyer KA, et al. Dietary Cholesterol and Cardiovascular Risk: A Science Advisory From the American Heart Association. Circulation [Internet]. 2020;141(3). doi: 10.1161/CIR.0000000000000743.

26. Pinckard K, Baskin KK, Stanford KI. Effects of Exercise to Improve Cardiovascular Health. Front Cardiovasc Med. 2019;6:69. doi: 10.3389/ fcvm.2019.00069.

27. Savla JJ, Levine BD, Sadek HA. The Effect of Hypoxia on Cardiovascular Disease: Friend or Foe? High Alt Med Biol. 2018;19(2):124-30. doi: 10.1089/ham.2018.0044

28. Vetter TR, Jesser CA. Fundamental Epidemiology Terminology and Measures: It Really Is All in the Name. Anesth Analg. 2017;125(6):214651. doi: 10.1213/ANE.0000000000002554.

29. Kuller LH. Epidemiology: Then and Now. Am J Epidemiol. 2016;183(5):372-80. doi: 10.1093/aje/kwv158.

30. Solomon SD, Pfeffer MA. The Future of Clinical Trials in Cardiovascular Medicine. Circulation. 2016;133(25):2662-70. doi: 10.1161/ CIRCULATIONAHA.115.020723. 